International Journal of Pure and Applied Mathematics

Volume 83 No. 1 2013, 45-53

ISSN: 1311-8080 (printed version); ISSN: 1314-3395 (on-line version)

url: http://www.ijpam.eu

doi: http://dx.doi.org/10.12732/ijpam.v83i1.5

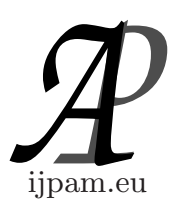

\title{
CLOSED GEODESIC LENGTHS IN HYPERBOLIC LINK COMPLEMENTS IN $S^{3}$
}

\author{
Sreekrishna Palaparthi \\ Department of Mathematics \\ IIT Guwahati \\ PIN 781039, Guwahati, Assam, INDIA
}

\begin{abstract}
Adams and Reid produced an upper bound for the length of a shortest closed geodesic in a hyperbolic knot or link complement in closed 3manifolds which do not admit any Riemannian metric of negative curvature. We demonstrate that the length of an $n^{\text {th }}$ shortest closed geodesic in such manifolds is also bounded above for $n>1$ and produce explicit upper bound on this length.
\end{abstract}

AMS Subject Classification: 57M25

Key Words: hyperbolic knots, hyperbolic links, geodesics, closed geodesics, length spectrum, lengths of geodesics

\section{Introduction}

A systole in a Riemannian manifold is a shortest closed geodesic, if one exists. Adams, Hass and Scott [1] showed that every hyperbolic 3-manifold has a shortest closed geodesic. Adams and Reid [2] used the $2 \pi$-theorem of Thurston and Gromov to produce an upper bound of 7.35534.. on the systole length for a hyperbolic link complement in $S^{3}$. Since, Agol [4] and Lackenby [9] showed that the bound of $2 \pi$ in the $2 \pi$-theorem could be improved to 6 and that this bound is sharp. This lowers the upper bound on the systole length for a hyperbolic

Received: June 27, 2012

(c) 2013 Academic Publications, Ltd. url: www.acadpubl.eu 
link complement in $S^{3}$ to $7.171646 \ldots$ Generalizing the result of Adams and Reid [2], we give an upper bound on the length of a geodesic of a hyperbolic knot complement in $S^{3}$ as a function of its length rank. In particular we prove:

Theorem 1. Let $M$ be a closed 3-manifold which does not admit any Riemannian metric of negative curvature. Let $L$ be a link in $M$, whose complement in $M$ admits a complete hyperbolic metric of finite volume. Then the length of the $n^{\text {th }}$ shortest closed geodesic in $M$ is bounded above by $\operatorname{Re}\left(2 \cosh ^{-1}(1+18 i(n+1))\right)$.

\section{Upper Bound on The Length of a Systole}

The $2 \pi$-theorem of Gromov and Thurston gives bounds on exceptional Dehn filling curve lengths. Here is the statement of the $2 \pi$-theorem:

Theorem 2. (The $2 \pi$-theorem) Let $M$ be a cusped hyperbolic 3-manifold with $n$ cusps. Let $T_{1}, . ., T_{n}$ be disjoint cusp tori for the $n$ cusps of $M$, and $r_{i}$ a slope on $T_{i}$ represented by a geodesic $a_{i}$ whose length in the Euclidean metric on $T_{i}$ is greater than $2 \pi$, for each $i=1 \ldots n$. Then $M\left(r_{1}, . ., r_{n}\right)$ admits a metric of negative curvature.

Agol [4] and Lackenby [9] independently improved the bound in the $2 \pi$ theorem to 6 . Here is a version of the theorem as stated in Lackenby [9]:

Theorem 3. (see [9], Theorem 4.9) Let $M$ be a compact orientable 3manifold with interior having a complete finite volume hyperbolic structure. Let $s_{1}, . ., s_{n}$ be slopes on $\partial M$, with one $s_{i}$ on each component of $\partial M$. Suppose that there is a horoball neighbourhood $N$ of the cusps of $M-\partial M$ on which each $s_{i}$ has length more than 6. Then, the manifold obtained by Dehn filling along $s_{1}, . ., s_{n}$ is irreducible, atoroidal and not Seifert fibred, and has infinite, word hyperbolic fundamental group.

Agol [4] called a manifold satisfying the conclusions of the above theorem hyperbolike. By Perelman's proof of the Thurston's geometrization conjecture (which appeared later), a hyperbolike 3-manifold admits a metric of negative curvature. Agol [4] produced examples of hyperbolic 3-manifolds to show that the bound of 6 in this theorem is sharp.

Let $M$ be a closed manifold which does not admit any metric of negative curvature and let $K$ be a knot in $M$ such that $M-K$ admits a complete hyperbolic metric of finite volume. Dehn filling $M-K$ along the meridian curve of the knot $K$ gives back $M$. So by Theorem 3, this meridian curve is 
at-most 6 in length. Adams and Reid [2] used this bound on the meridian to produce a bound on the length of the shortest closed geodesic in $M-K$. They do so by considering the geodesic in the class of the loop which is a product of two meridians. In the event that the product is a peripheral loop, there is an embedded twice punctured disk in the manifold, which gives the bound on the length of the systole.

Let $N$ be a cusped hyperbolic 3-manifold. Fix a cusp $C$ in $N$. The pre-image of a horoball neighborhood of the cusp $C$ is a disjoint union of horoballs in $\mathbb{H}^{3}$, which can be expanded equivariantly until the first two touch. The projection of this configuration to $N$ is called a maximal cusp in $N$. The waist size of a cusp is the length of the shortest essential simple closed curve corresponding to a parabolic isometry on a maximal cusp torus associated to that cusp.

In the case of manifolds with more than one cusp we can expand the horoballs corresponding to various cusps equivariantly until the first two horoballs touch and consider the projection of such a configuration to the manifold $N$. The point of tangency in this case need not necessarily be of a cusp with itself, it could potentially be a point of tangency of two different cusps.

We shall use the upper half-space model of $\mathbb{H}^{3}$ and always assume that $\infty$ and 0 in $\partial \mathbb{H}^{3}$ are the parabolic fixed points of the fundamental group of any cusped hyperbolic 3-manifold which occurs anywhere in this discussion. As a result, the point of tangency, say $p$, of the horoballs centred at 0 and $\infty$ will have co-ordinates $(0,0, t)$ for some $t>0$. In the pre-image of the maximal cusp, the boundary of the horoball centred at $\infty$ is a Euclidean plane parallel to the $x-y$-plane and will be denoted by $\mathscr{H}$ and any other horoball which is tangent to the horoball centred at $\infty$ is called a full-sized horoball. The full-sized horoball centred at 0 will be denoted by $\mathscr{H}_{0}$.

At this point we will have one degree of freedom left to further conjugate the fundamental group of a cusped hyperbolic 3-manifold inside $P S L(2, \mathbb{C})$. If the group is conjugated so that the horosphere $\mathscr{H}$ lies at the height $z=1$ and $p$ has co-ordinates $(0,0,1)$, then this normalization of the maximal cusp will be called standard form, as mentioned in [2]. If we do not choose this normalization, then we still have one degree of freedom left to conjugate the fundamental group of the cusped hyperbolic 3-manifold under consideration.

The main theorem of Adams and Reid [2] for hyperbolic knot or link complements in a closed oriented manifold $M$ which does not admit any metric of negative curvature is stated here.

Theorem 4. (see [2], Theorem 1.1) Let $M$ be closed orientable 3-manifold which does not admit any Riemannian metric of negative curvature. Let $L \subset M$ be a link in $M$ whose complement admits a complete hyperbolic structure of 
finite volume. Then $\mathscr{L}_{1}(M-K) \leq 7.35534$..

Using Theorem 3 this bound can be improved to 7.171646.

\section{Upper Bounds on $\mathscr{L}_{n}(M-K)$}

In this section we will state and prove Theorem 1. First, we begin with proving the following variant of the theorem and later improve the bounds in this variant.

Theorem 5. Let $M$ be a closed orientable 3-manifold which does not admit any Riemannian metric of negative curvature. Let $K$ be a knot in $M$, whose complement in $M$ admits a complete hyperbolic structure of finite volume. Then the length of the second shortest closed geodesic in the manifold $M-K$ is bounded above by 24 .

Proof. Let $\Gamma=\pi_{1}(M-K)$. With a slight abuse of notation we identify $\Gamma$ with a discrete subgroup of $P S L(2, \mathbb{C})$.

Let $x_{1}$ be a lift of a meridian curve of $K$ to $\mathscr{H}$ starting at the point $p$ and ending at another point, say $q$. The points $p$ and $q$ project to the tangency point in the maximal cusp torus in $M-K$. Let $\gamma_{1} \in \Gamma$ be the parabolic isometry which corresponds to the projection of $x_{1}$. The parabolic fixed point of $\gamma_{1}$ will then be $\infty$. Let $x_{0}$ be a lift of a meridian curve of $K$ to $\mathscr{H}_{0}$, starting at the point $p$. Let $\gamma_{0} \in \Gamma$ be the parabolic element determined by the projection of $x_{0}$. The parabolic fixed point of $\gamma_{0}$ will be 0 . For a positive integer $n$, let $x_{n+1}=\gamma_{1}^{n}\left(x_{1}\right)$ i.e. $x_{n+1}$ is the translation of $x_{1}$ via the parabolic isometry $\gamma_{1}^{n}$, so that $x_{n+1}$ is also a lift of a meridian to $\mathscr{H}$, but based at the point $\gamma_{1}^{n}(p)$. For a positive integer $n$ consider the path $x=x_{n}^{-1} x_{n-1}^{-1} \ldots x_{1}^{-1} x_{0}$. This path $x$ projects to a loop in $M-K$. In fact, the projection of $x$ is the element $\gamma_{0} \cdot \gamma_{1}^{-n}$ of $\Gamma$.

Since 0 and $\infty$ are chosen to be the parabolic fixed points of $\Gamma$, we have one more degree of freedom to conjugate $\Gamma$ within $P S L(2, \mathbb{C})$. We can conjugate $\Gamma$ so as to have the representation of $\gamma_{1}$ in $\operatorname{PSL}(2, \mathbb{C})$ as the element $\mathbf{A}=\left(\begin{array}{ll}1 & 2 \\ 0 & 1\end{array}\right)$ In geometrical terms, $\gamma_{1}$ can be thought of as acting with a fixed point at $\infty$ and translating every horosphere centred at $\infty$ (i.e. every Euclidean plane parallel to the $x-y$-plane in the upper half-space model) along the $x$-axis by 2 units. This arrangement of the maximal cusp will be called the altered standard form of normalization of the cusp. 
Fix the altered standard form of the maximal cusp. Then, the representation of $\gamma_{0}$ in $\operatorname{PSL}(2, \mathbb{C})$ will be a matrix $\mathbf{B}=\left(\begin{array}{ll}1 & 0 \\ r & 1\end{array}\right)$ and that of an element $\gamma_{0} \cdot \gamma_{1}^{-n}$, for a given positive integer $n$, will be a matrix

$$
\mathbf{C}_{\mathbf{n}}=\left(\begin{array}{rr}
1 & -2 n \\
r & 1-2 r n
\end{array}\right)
$$

for some $r \in \mathbb{C}$ and $r \neq 0$. So the trace of an element $\gamma_{0} \cdot \gamma_{1}^{-n}$ is $2-2 r n$.

Let $\mathbf{S}=\left\{\gamma_{0} \cdot \gamma_{1}^{-n}: n \in \mathbb{N}\right\}$ We can draw two conclusions about the elements in this set based on their traces. Let $\gamma_{0} \cdot \gamma_{1}^{-m}$ and $\gamma_{0} \cdot \gamma_{1}^{-n}$ be two different elements of $\mathbf{S}$ for some positive integers $m$ and $n$. Since conjugation in $P S L(2, \mathbb{C})$ preserves traces upto sign, these two elements are conjugate only if $2-2 r m=-(2-2 r n)(r \neq 0$ implies that $2-2 r m \neq 2-2 r n)$. But then $2-2 r m=2 r n-2$ would imply that $r=\frac{2}{m+n}$ and hence real, in which case the trace of $\gamma_{0} \cdot \gamma_{1}^{-1}$ is $2-2 r=2-\frac{4}{l+m}<2$, which is impossible as $\gamma_{0} \cdot \gamma_{1}^{-1}$ cannot be elliptic ( $\Gamma$ is a torsion-free Kleinian group since $M-K$ is a manifold). So two different elements of $\mathbf{S}$ cannot be conjugate in $\Gamma$. This is the first conclusion. For $n>1$ an element $\gamma_{0} \cdot \gamma_{1}^{-n}$ can be parabolic only if $2-2 r n=-2(2-2 r n$ cannot be 2 since $r \neq 0$ ), which would require that $r=\frac{2}{n}$. But this would once again imply that the trace of $\gamma_{0} \cdot \gamma_{1}^{-1}$ is $2-2 r=2-\frac{2}{n}<2$. So the elements of $\mathbf{S}$ are all loxodromic elements for $n>1$. This is the second conclusion.

Now the length of the second shortest closed geodesic in $M-K$ is bounded above by the length of the geodesic belonging to the class of $\gamma_{0} \cdot \gamma_{1}^{-2}$, unless the shortest closed geodesic in $M-K$ is the geodesic belonging to the class of $\gamma_{0} \cdot \gamma_{1}^{-2}$, in which case, the length of the second shortest closed geodesic in $M-K$ is bounded above by the length of the geodesic belonging to the class of $\gamma_{0} \cdot \gamma_{1}^{-3}$. So in any case, the second shortest closed geodesic in the manifold $M-K$ is at-most 24 in length proving the theorem.

The following result gives us a way to reduce the bound of the above theorem further.

Theorem 6. Let $N$ be a finite-volume hyperbolic 3-manifold with at-least one cusp. Let a maximal cusp torus have a non-trivial curve $c$ corresponding to a parabolic isometry of length $w$. Let $n \in \mathbb{N}$ Then

$$
\mathscr{L}_{n}(N) \leq \operatorname{Re}\left(2 \cosh ^{-1}\left(\left(2+i(n+1) w^{2}\right) / 2\right)\right)
$$

Proof. We start with a setup very similar to the one in the proof of Theorem 5 . Let $\Gamma=\pi_{1}(N)$ with a basepoint * being the point of tangency of the maximal 
cusp in $N$. We can arrange so that the maximal cusp is in the standard form and so that $p$ with co-ordinates $(0,0,1)$ is in the pre-image of * when we lift $N$ to $\mathbb{H}^{3}$. Let $x_{1}$ be a lift of the curve $c$ to $\mathscr{H}$ starting at the point $p$ and ending at another point, say $q$. The points $p$ and $q$ project to the tangency point in the maximal cusp torus in $N$. Let $\gamma_{1} \in \Gamma$ be the parabolic isometry which corresponds to the projection of $x_{1}$. Let $x_{0}$ be a lift of the curve parallel to $c$ to $\mathscr{H}_{1}$, starting at the point $p$. Let $\gamma_{0} \in \Gamma$ be the parabolic element determined by the projection of $x_{0}$. For a positive integer $n$, let $x_{n+1}=\gamma_{1}^{n}\left(x_{1}\right)$, so that $x_{n+1}$ is also a lift of a curve parallel to $c$ to $\mathscr{H}$, but based at the point $\gamma_{1}^{n}(p)$. For a positive integer $n$ the path $x=x_{n}^{-1} x_{n-1}^{-1} \ldots x_{1}^{-1} x_{0}$ projects to a loop in $N$ which is the element $g_{n}=\gamma_{0} \cdot \gamma_{1}^{-n}$ of $\Gamma$. If needed, by replacing $x_{0}$ by $x_{0}^{-1}$, we can be sure that the angle between $x_{1}$ and $x_{0}$ at $p$ is at-most $\pi / 2 . \gamma_{1}^{-1}$ and $\gamma_{2}$ can be represented by the matrices

$$
\mathbf{A}=\left[\begin{array}{cc}
1 & w \\
0 & 1
\end{array}\right] \text { and } \mathbf{B}=\left[\begin{array}{ll}
1 & 0 \\
r & 1
\end{array}\right]
$$

respectively where $r \in \mathbb{C}$ is some constant with $r \neq 0$, and for a given positive integer $n, g_{n}$ gets a representation

$$
\mathbf{C}_{\mathbf{n}}=\left[\begin{array}{rr}
1 & n w \\
r & 1+r n w
\end{array}\right]
$$

So the trace of an element $\mathbf{C}_{\mathbf{n}}$ is $2+r n w$. For $n>12+r n w=-2$ would imply that the trace of $\mathbf{C}_{1}$ is $2-r w=2-\frac{4}{n}$. But since $0 \leq 2-\frac{4}{n}<2$, this would imply that there are elliptic elements in $N$ which is impossible. So for $n>1, g_{n}$ has to be a loxodromic element. It is easy to see that the maximum length of the invariant geodesic axis of $g_{n}$, for any given $n$ occurs when the absolute value of the angle between $x_{0}$ and $x_{1}$ at $p$ is exactly $\frac{\pi}{2}$ so that $r$ can be taken to be $i w$ so that the trace of $\mathbf{C}_{\mathbf{n}}$ is $2+i n w^{2}$. By the standard identities relating the trace to translation length, the geodesic in the class of $g_{n}$ is at most $R e 2 \cosh ^{-1}\left(\left(2+i n w^{2}\right) / 2\right)$. But as in the proof of Theorem 5 when the geodesic in the class of $g_{2}$ is a shortest closed geodesic in the manifold, we might need to use $g_{3}$ to bound $\mathscr{L}_{2}(N)$. So in this special case there is a cascading effect and so we might need to use the length bound on the geodesic representative of $g_{n+1}$ instead of $g_{n}$ to bound $\mathscr{L}_{n}(N)$. In any case $\mathscr{L}_{n}(N)$ is not more than $\operatorname{Re} 2 \cosh ^{-1}\left(\left(2+i(n+1) w^{2}\right) / 2\right)$.

This theorem gives an improvement over the bounds in Theorem 5. For a closed 3-manifold $M$ which does not admit any Riemannian metric of negative curvature and a hyperbolic knot $K$ in it, we can take $w=6$ to obtain an upper bound on $\mathscr{L}_{n}(M-K)$. We then have: 
Corollary 7. Let $M$ be a closed 3-manifold which does not admit any Riemannian metric of negative curvature. Let $K$ be a knot in $M$, whose complement in $M$ admits a complete hyperbolic metric of finite volume. Then the length of the second shortest closed geodesic in the manifold $M-K$ is bounded above by 9.364776. . and $\mathscr{L}_{n}(M-K) \leq \operatorname{Re}\left(2 \cosh ^{-1}(1+18 i(n+1))\right)$.

Now we state and prove the main theorem which is about hyperbolic links in such manifolds $M$ as in Corollary 7 . We wish to emphasize that the proof of this theorem is the same as the proof for the systole length bound in hyperbolic link complements in such manifolds as $M$ which was given in [2]. Although it is the original authors' idea, we wish to provide the proof here with the required modification in the statement of the Theorem, for completeness.

Theorem 8. Let $M$ be a closed 3-manifold which does not admit any Riemannian metric of negative curvature. Let $L$ be a link in $M$, whose complement in $M$ admits a complete hyperbolic metric of finite volume. Then $\mathscr{L}_{n}(M-L)$ is bounded above by $\operatorname{Re}\left(2 \cosh ^{-1}(1+18 i(n+1))\right)$.

Proof. In a collection of all disjoint cusps in $M-L$, there should be at-least one cusp with a shortest non-trivial loop whose length is at-most 6 by Theorem 3. So in a collection of all the cusps in $M-L$ first suppose that there is only one cusp with a shortest non-trivial loop $c$ whose length is at-most 6 . Now start expanding this cusp $C$ equivariantly. If this cusp expands to a maximal cusp while its waist size does not exceed 6 , then we can use the arguments of Theorems 5 and 6 to obtain the stated result. Otherwise the waist size of $C$ exceeds 6 before it becomes maximal. But then in order that the collection of all the cusps have at-least one cusp with waist length at most 6 , at some point (in time) during its expansion $C$ should have been tangent to another cusp, say $C_{1}$, before $C$ 's waist length exceeds 6 and should have caused $C_{1}$ to contract as it expands beyond that point (in time). At the point (in time) where $C$ first becomes tangent to $C_{1}$, the waist length of either of these cusps should have been at-most 6 . So at this point in time we can take a lift of $M-L$ to $\mathbb{H}^{3}$ such that the cusp $C$ lifts to a collection of horoballs where one of them is centred at $\infty$ and the cusp $C_{1}$ lifts to a collection of horoballs such that one of these is centred at 0 and use lifts of meridians of $C$ onto $\mathscr{H}$ and lift of a meridian of $C_{1}$ onto $\mathscr{H}_{0}$ and argue as in theorem 6 .

Now suppose that there are two or more cusps in the collection of all the cusps of $M-L$ whose waist size is at-most 6 . Pick one and expand it. If it expands to a maximal cusp while its waist length is still at most 6 or if it bumps into another when the waist size of either of these cusps is less than 6 , then argue as before. But if neither happens, then delete this cusp from 
consideration and take the collection of cusps without this one and start the argument again. Eventually there will be a cusp which will have its maximal cusp waist size at most 6 or will be tangent to another cusp at some point during its expansion such that the waist size of either will be less than 6 and we argue as before. This proves the theorem.

\section{References}

[1] Colin Adams, Joel Hass, Peter Scott, Simple closed geodesics in hyperbolic 3-manifolds, Bull. London Math. Soc., 31, No. 1 (1999), 81-86.

[2] Colin C. Adams, Alan W. Reid, Systoles of hyperbolic 3-manifolds, Math. Proc. Cambridge Philos. Soc., 128, No. 1 (2000), 103-110.

[3] Colin Adams, Hyperbolic knots, In: Handbook of Knot Theory, Elsevier B.V., Amsterdam (2005), 1-18.

[4] Ian Agol, Bounds on exceptional Dehn filling, Geom. Topol., 4 (2000), 431-449, Electronic.

[5] Riccardo Benedetti, Carlo Petronio, Lectures on Hyperbolic Geometry, Universitext, Springer-Verlag, Berlin (1992), xiv+330.

[6] Ted Chinburg, Alan W. Reid, Closed hyperbolic 3-manifolds whose closed geodesics all are simple, J. Differential Geom., 38, No. 3 (1993), 545-558.

[7] Sally M. Kuhlmann, Geodesic knots in cusped hyperbolic 3-manifolds, Algebr. Geom. Topol., 6 (2006), 2151-2162, Electronic.

[8] Sally Kuhlmann, Geodesic knots in closed hyperbolic 3-manifolds, Geom. Dedicata, 131 (2008), 181-211.

[9] Marc Lackenby, Word hyperbolic Dehn surgery, Invent. Math., 140, No. 2 (2000), 243-282.

[10] Marc Lackenby, Darren D. Long, Alan W. Reid, Covering spaces of arithmetic 3-orbifolds, Int. Math. Res. Not. IMRN, No. 12 (2008), Art. ID rnn036.

[11] Colin Maclachlan, Alan W. Reid, The Arithmetic of Hyperbolic 3Manifolds, Graduate Texts in Mathematics, 219, Springer-Verlag, New York (2003), xiv+463. 
[12] A. Marden, Outer Circles. An Introduction to Hyperbolic 3-Manifolds, Cambridge University Press, Cambridge (2007), xviii +427.

[13] Sally M. Miller, Geodesic knots in the figure-eight knot complement, Experiment. Math., 10, No. 3 (2001), 419-436.

[14] John G. Ratcliffe, Foundations of Hyperbolic Manifolds, Graduate Texts in Mathematics, 149, Springer-Verlag, New York (1994), xii+747.

[15] William P. Thurston, The Geometry and Topology of 3-Manifolds, Princeton University Lecture Notes (1978). 
